\title{
Calidad de vida en adultos mayores con enfermedad pulmonar obstructiva crónica
}

\section{Quality of life in older adults with chronic obstructive pulmonary disease}

\section{Qualidade de vida em idosos com doença pulmonar obstrutiva crônica}

Diana Isabel Cáceres-Rivera, Enf., MSc., PhD.*

Zayne Milena Roa-Díaz, Enf., MSc.**

Clara Lucia Domínguez, Enf.***

Javier Hernán Carreño-Robayo, FT., Esp.****

Mauricio Ariel Orozco-Levi, MD., Esp., PhD.*****

\section{Resumen}

Introducción: La enfermedad pulmonar obstructiva crónica es una importante causa de mortalidad y morbilidad a nivel mundial y tiene efectos psicosociales y fisiopatológicos que repercuten de manera significativa en la calidad de vida de quienes la padecen. Objetivo: Evaluar la calidad de vida de pacientes con enfermedad pulmonar obstructiva crónica a través del instrumento SF-36, versión en español para Colombia (SF-36 v2), en un servicio de consulta externa de Floridablanca, Colombia, durante el año 2015. Metodología: Estudio de corte transversal descriptivo realizado en 40 pacientes con diagnóstico de enfermedad pulmonar obstructiva crónica que asistieron al servicio de consulta externa por Neumología entre los meses de febrero y noviembre de

* Enfermera, Magíster en Enfermería, Doctorado en Biomedicina. Servicio de Neumología, Unidad de Diseño y Desarrollo de Investigaciones, Fundación Cardiovascular de Colombia. Facultad de Enfermería, Universidad Cooperativa de Colombia, Bucaramanga, Santander, Colombia.

** Enfermera, Magíster en Epidemiología. Unidad de Diseño y Desarrollo de Investigaciones, Fundación Cardiovascular de Colombia. Escuela de Enfermería, Universidad Industrial de Santander, Bucaramanga, Santander, Colombia.

*** Enfermera. Unidad de Diseño y Desarrollo de Investigaciones, Fundación Cardiovascular de Colombia, Floridablanca, Santander, Colombia.

**** Fisioterapeuta, Especialista en Ejercicio Físico para la Salud. Servicio de Neumología, Unidad de Diseño y Desarrollo de Investigaciones, Fundación Cardiovascular de Colombia. Dirección Médica de Investigaciones, Desarrollo e Innovación Tecnológica. Fundación Oftalmológica de Santander, Floridablanca, Santander, Colombia.

***** Médico, Especialista en Neumología, Doctorado en Biomedicina. Servicio de Neumología, Unidad de Diseño y Desarrollo de Investigaciones, Fundación Cardiovascular de Colombia, Floridablanca, Santander, Colombia.

Correspondencia: Diana Isabel Cáceres Rivera. Calle 155 $5^{\mathrm{a}}$ No. 23-58, Urbanización El Bosque, Floridablanca, Colombia. Celular: E-mail: dianacaceresrivera@gmail.com. 
2015. El cuestionario SF-36 v2 fue diligenciado por todos los participantes; previamente, se firmó el consentimiento informado. La base de datos fue digitada, bajo licencia, en QualityMetric Health Outcomes ${ }^{\mathrm{TM}}$ Scoring Software 4.0. Resultados: Participaron 40 pacientes, con una edad media de 73 ( $\mathrm{DE} \pm$ 8.4) años. En las dimensiones de funcionamiento físico se encontraron valores inferiores a 50; por otra parte, las dimensiones Funcionamiento Social y Rol Emocional presentaron valores superiores y cercanos a los reportados por población sana. Conclusiones: Los pacientes con enfermedad pulmonar obstructiva crónica mostraron una disminución en la calidad de vida comparado con los valores de referencia en población en general en sus dos componentes: físico y mental. Se ratifica el impacto que tiene esta enfermedad en todas las subescalas que componen este constructo, especialmente en el componente físico. [Cáceres-Rivera DI, Roa-Díaz ZM, Domínguez CL, Carreño-Robayo JH, Orozco-Levi $M A$. Calidad de vida en adultos mayores con enfermedad pulmonar obstructiva crónica. MedUNAB. 2018;21(1):4658. doi: 10.29375/01237047.2512].

Palabras clave: Enfermedad Pulmonar Obstructiva Crónica; Calidad de vida; Encuestas y Cuestionarios; Anciano; Colombia.

\section{Abstract}

Introduction: Chronic obstructive pulmonary disease is one the most significant causes of mortality and morbidity worldwide. It has psychosocial and pathophysiological effects that impact in a significant way to those who suffer from it. Objective: To evaluate quality of life from patients suffering from chronic obstructive pulmonary disease through the SF-36 survey (Spanish version for Colombia (SF-36 v2)) made during an external medical appointment made in Floridablanca, Colombia in 2015. Methodology: Descriptive cross sectional study performed to 40 patients diagnosed with chronic obstructive pulmonary disease. These patients attended to external pulmonology medical appointments between February and November of 2015 and filled out the short form SF-36 v2 after signing informed consent. The database was created under license using QualityMetric Health Outcomes ${ }^{\mathrm{TM}}$ Scoring Software 4.0. Results: 40 patients, with an average age of 73 years $(\mathrm{DE} \pm 8.4)$, participated in this study. When analyzing data, it was found that the physical functioning dimensions were values lower than 50. On the other hand, it was found that dimensions related to social functioning and emotional role had higher values and close to the ones reported by healthy population. Conclusions: Patients diagnosed with chronic obstructive pulmonary disease showed a decrease in quality of life, in both of its dimensions: physical and mental, compared to healthy population's reference values. It is ratified that this disease impacts all sub-scales of this construct, specially the ones related to the physical component. [Cáceres-Rivera DI, Roa-Díaz ZM, Domínguez CL, Carreño-Robayo JH, Orozco-Levi MA. Quality of life in older adults with chronic obstructive pulmonary disease. MedUNAB. 2018;21(1):46-58. doi: 10.29375/01237047.2512].

Keywords: Pulmonary Disease, Chronic Obstructive; Quality of Life; Surveys and Questionnaires; Aged; Colombia.

\section{Resumo}

Introdução: A doença pulmonar obstrutiva crônica é uma importante causa de mortalidade e morbidade em todo o mundo e tem efeitos psicossociais e fisiopatológicos que têm um impacto significativo na qualidade de vida daqueles que sofrem com ela. Objetivo: Avaliar a qualidade de vida de pacientes com doença pulmonar obstrutiva crônica através do instrumento SF-36, versão em espanhol para a Colômbia (SF-36 v2), em um serviço ambulatorial de Floridablanca, Colômbia, durante 2015. Metodologia: Estudo descritivo de corte transversal feito em 40 pacientes com diagnóstico de doença pulmonar obstrutiva crônica que compareceram ao ambulatório de Pneumologia entre os meses de fevereiro e novembro de 2015. O questionário SF-36 v2 foi preenchido por todos os participantes; anteriormente, o consentimento informado foi assinado. $\mathrm{O}$ banco de dados foi digitado, sob licença, em Quality Metric Health Outcomes ${ }^{\mathrm{TM}}$ Scoring Software 4.0. Resultados: Participaram 40 pacientes, com média de idade de 73 (DE \pm 8.4) anos. Nas dimensões do funcionamento físico, valores menores que 50 foram encontrados; por outro lado, as dimensões do Funcionamento Social e do Papel Emocional apresentaram valores superiores e próximos aos relatados pela população saudável. Conclusões: Os pacientes com doença pulmonar obstrutiva crônica apresentaram diminuição da qualidade de vida em relação aos valores de referência na população geral em seus dois componentes: físico e mental. O impacto desta doença em todas as subescalas que compõem este construto, especialmente no componente físico, é confirmado. [Cáceres-Rivera DI, Roa-Díaz ZM, Domínguez CL, Carreño-Robayo JH, Orozco-Levi MA. Qualidade de vida em idosos com doença pulmonar obstrutiva crônica. MedUNAB. 2018;21(1):4658. doi:10.29375/01237047.2512].

Palavras-chave: Doença Pulmonar Obstrutiva Crônica; Qualidade de Vida; Inquéritos e Questionários; Idoso; Colômbia. 


\section{Introducción}

La iniciativa global para la enfermedad pulmonar obstructiva crónica (GOLD, por sus siglas en inglés) define la enfermedad pulmonar obstructiva crónica (EPOC) como una enfermedad común, prevenible y tratable, caracterizada por una limitación persistente del flujo de aire que es usualmente progresiva. Se produce una respuesta inflamatoria crónica en los pulmones y las vías aéreas, asociada a la exposición a partículas nocivas o gases. Esta enfermedad es una importante causa de mortalidad y morbilidad y se prevé que, para el año 2020, ocupará el tercer lugar entre las principales enfermedades a nivel mundial, de acuerdo con lo estimado por la Organización Mundial de la Salud (1).

La prevalencia de la EPOC alrededor del mundo varía considerablemente. No obstante, la información obtenida de diversos estudios sugiere una mayor prevalencia en la población masculina, adultos mayores de 40 años y en fumadores o ex-fumadores. En Latinoamérica, el estudio PLATINO, realizado en Chile, Brasil, Uruguay, México y Venezuela, reportó una prevalencia del $15 \%$ del diagnóstico de EPOC en personas mayores de 40 años (2). En Colombia, a pesar de la poca información disponible sobre la epidemiología de la EPOC, se sabe que la prevalencia y la magnitud de sus consecuencias varían ampliamente por regiones, tal y como lo demostró el estudio PREPOCOL (Prevalencia de la EPOC en Colombia). Este estudio determinó, para el 2005, que 9 de cada 100 personas mayores de 40 años padecían esta enfermedad, porcentaje que se distribuyó principalmente en Medellín (13.5\%), Bogotá y Cali $(8.5 \%)$, seguido de Bucaramanga (7.9\%) y Barranquilla (6.2\%) (3).

Es importante destacar que esta patología es también reconocida por el gran impacto que tiene sobre los costos en los distintos sistemas de salud, debido a la presencia de exacerbaciones que requieren de manejo intrahospitalario (4). En los países europeos, la EPOC representa el 56\% (38.6 billones de euros) de los costos por enfermedades respiratorias. Por su parte, en Estados Unidos, los costos directos estimados de la EPOC son de 29.5 mil millones y los indirectos de 20.4 mil millones de dólares. Estos costos son frecuentemente estimados a través de los DALYs (Dissability Ajusted Life-Year), en su traducción al español se conocen como la "suma de años perdidos a causa de incapacidad ajustados por la severidad de la enfermedad". En adición a lo anterior, la EPOC no solo afecta al individuo enfermo, sino también a los miembros de la familia, pues, alguno de ellos asume un nuevo rol de cuidador y renuncia a sus actividades laborales, lo que llega a producir una situación de riesgo para la economía de una nación (5).

Lo anterior destaca la importancia de incluir en el manejo de estos pacientes no solo el alivio de los síntomas y la mejora del estado funcional, sino también cambios en la calidad de vida $(6,7)$. En relación con este tema, se sabe que tanto los efectos psicosociales como fisiopatológicos de la EPOC alteran de manera significativa la calidad de vida de quienes la padecen (8-10).

Si bien el concepto de calidad de vida (CV) es complejo, global, inclusivo y multidisciplinario (11), fue definido por la Organización Mundial de la Salud como la estimación personal de un individuo de su situación en la vida, dentro del contexto cultural, socioeconómico y de valores en que vive, así como en relación con sus objetivos, expectativas, valores e intereses (12). Asimismo, en el área de la Salud este concepto ha tomado un significado propio y ha generado un constructo denominado calidad de vida relacionado con la salud (CVRS), que ha sido un indicador de evaluación de los efectos de la enfermedad y sus tratamientos en la vida de los pacientes. Se reconoce, así, el impacto de las patologías, su evolución, estadio clínico, entre otros factores $(13,14)$.

En cuanto a las dimensiones que abarca este constructo, hacia la década de los 80 se concibieron tres grandes enfoques: salud mental, funcionamiento físico y funcionamiento social (15). Posteriormente, este concepto evolucionó e incluyó atributos más detallados como los sentimientos de satisfacción con la vida en general, la capacidad mental para evaluar la vida propia, el estado de salud físico, mental, social, emocional y las condiciones de vida. Se puede decir que la literatura sobre CV concuerda fundamentalmente en tres asuntos: es subjetiva, el puntaje asignado a cada dimensión es diferente en cada persona y el valor asignado a cada dimensión puede cambiar a través de la vida (16).

Existen diversos instrumentos para realizar la medición de CVRS en los pacientes con enfermedades crónicas como la EPOC (17). A través de ellos, se ha identificado un empobrecimiento de las actividades diarias y un progresivo o permanente acortamiento de las propias posibilidades, condiciones que deterioran el concepto de sí mismo y el sentido de la vida y contribuyen a la aparición de estados depresivos (18). 
El Short Form (SF-36) es un cuestionario general que se puede aplicar para medir la CVRS en grupos con diferentes edades, enfermedades o tratamientos. Por esta razón, el Instituto de Salud del New England Medical Center lo incluyó, en 1991, en el proyecto Quality of Life Assessment (IQUOLA), en el que se obtuvieron versiones validadas en diferentes idiomas, incluyendo el español (19). Este cuestionario consta de 36 preguntas con 8 subescalas que agrupan tópicos como salud funcional, bienestar, dos escalas psicométricas para salud mental y física y un ítem para concepto de la propia salud (20).

Teniendo en cuenta la importancia que tiene para Enfermería conocer sobre la calidad de vida en materia de salud de pacientes crónicos, el objetivo de este estudio fue evaluar la calidad de vida a través del SF-36 v2 en español para Colombia, en los pacientes con diagnóstico de EPOC que asistieron al servicio de neumología de una institución de alta complejidad en Colombia durante el 2015.

\section{Metodología}

Se realizó un estudio de corte transversal, con muestreo a conveniencia, en el periodo comprendido entre febrero y noviembre de 2015 , en personas que acudieron al programa de rehabilitación pulmonar del servicio de neumología de una institución de alta complejidad en Colombia. El criterio de inclusión fue el diagnóstico confirmado de EPOC, de acuerdo con los parámetros establecidos por la iniciativa mundial GOLD: reducción del cociente entre el volumen espiratorio forzado en el primer segundo $\left(\mathrm{FEV}_{1}\right)$ y la capacidad vital forzada (FVC), en una prueba pos broncodilatadora con un valor inferior a 0.70 . Se excluyeron aquellos pacientes que, por condiciones neurológicas o físicas, no pudieran realizar las pruebas de valoración.

\section{Calidad de vida e instrumento SF-36}

Es una escala genérica que proporciona un perfil del estado de salud y es aplicable tanto a los pacientes, como a la población general. Ha resultado útil para evaluar la calidad de vida relacionada con la salud (CVRS) en la población general, o en subgrupos específicos, y comparar la carga de diversas enfermedades. Para este instrumento, se han descrito propiedades psicométricas adecuadas, evaluadas en más de 400 artículos publicados en el ámbito mundial. Estos permiten la comparación de resultados y lo convierten así en un instrumento con gran potencial en el campo de la CVRS (21).
El SF-36 está compuesto por 36 preguntas divididas en dos compontes, físico y mental, con ocho subescalas, así: diez preguntas pertenecen al funcionamiento físico (función física); dos, al funcionamiento social (función social); cuatro, a las limitaciones en el rol, debido a problemas físicos (rol físico); tres indagan por limitaciones en el rol, debido a problemas emocionales (rol emocional); cinco se relacionan con la salud mental (salud mental); cuatro, con la vitalidad (energía/fatiga); dos se refieren al dolor (dolor corporal); cinco, a la percepción general de salud (salud general) y una, que no forma parte de ninguna dimensión, mide el cambio de la salud en el tiempo. Cada subescala se califica de 0 a 100; este último puntaje es el más deseable.

En el presente estudio se utilizó un instrumento en físico, con los 36 ítems del SF-36 propuestos en el software QualityMetric Health Outcomes ${ }^{\mathrm{TM}}$ Scoring Software 4.0, en su versión en español para Colombia (22).

\section{Recolección y procesamiento de la información}

Una enfermera entrenada dio las indicaciones a cada uno de los participantes para diligenciaran el SF-36 v2 en español. La base de datos fue digitada, bajo licencia, en el software QualityMetric Health Outcomes $^{\mathrm{TM}}$ Scoring Software 4.0. Para la obtención de los puntajes en cada una de las dimensiones, el software realiza el siguiente proceso: a) cálculo de las puntuaciones de las ocho subescalas básicas; b) estandarización de las puntuaciones de cada subescala, restando la media poblacional de los valores anteriores y dividiendo por la desviación estándar (DE) poblacional; c) suma ponderada de los valores estandarizados; y d) transformación lineal, para obtener una distribución con un valor medio 50 y DE de 10 en la población general de referencia (22). Este proceso da ventajas en la interpretación de las puntuaciones, pues permite identificar un punto de corte de comparación con la calidad de vida en la población general y clasificar directamente los puntajes como mayores o menores que este valor (23). Por último, los datos fueron exportados para procesamiento en el programa Stata V14. Se realizó el cálculo de frecuencias absolutas y relativas para las variables cualitativas. Para las cuantitativas, medidas de tendencia central y de dispersión, de acuerdo al cumplimiento de supuestos de normalidad evaluados a través de prueba shapiro wilk.

El estudio contó con la aprobación del Comité de Ética de la Fundación Cardiovascular de Colombia. Todos 
los participantes firmaron una hoja de consentimiento, tras ser informados sobre las características del estudio, que se llevó a cabo siguiendo rigurosamente las recomendaciones éticas internacionales para investigación médica en humanos, según las normas recogidas en la Declaración de Helsinki. Acorde con la Resolución 008430 de 1993, este estudió se consideró de bajo riesgo. Igualmente, teniendo en cuenta el principio de confidencialidad planteado en la Ley 911 del 2004, se preservó la privacidad de los datos recolectados y la identidad de los participantes.

\section{Resultados}

La edad promedio de las 40 personas participantes en el estudio fue $73(\mathrm{DE} \pm 8.4)$ años; el $70 \%(\mathrm{n}=28)$ pertenecía al sexo masculino; el $82.5 \%(\mathrm{n}=33)$ residía en la ciudad de Bucaramanga. Con relación a la escolaridad, el $77.5 \%$ de los pacientes reportó un nivel de educación básica. Se confirmó tabaquismo en el $80 \%$ de ellos $(n=32)$; el $37.5 \%(n=15)$ presentó EPOC severo y, en una menor proporción, se encontró el diagnóstico de EPOC muy severo con un $12.5 \%(n=5)$. Se identificó una disminución de la PImax (Presión inspiratoria máxima) en el 55\% y una disminución de la PEmax (Presión espiratoria máxima) en el 22.5\% de los pacientes (Tabla 1).

En relación con las preguntas correspondientes a la percepción de la propia salud, el $50 \%(n=20)$ de los participantes la calificó, en general, como "regular", seguido de un 30\% (n=12) que la calificó como "buena". Al indagar por la percepción de su estado actual de salud, comparado con el percibido un año atrás, el 33\% (n=13) la consideró "algo peor", seguido de un $23 \%(\mathrm{n}=9)$ que señaló "algo de mejoría". En cuanto a preguntas relacionadas con la capacidad para realizar actividades de la vida diaria, se observó que un $70 \%$ expresó una gran limitación para la realización de actividades intensas, como levantar objetos pesados, y un 50\% para la realización de actividades moderadas, como mover una mesa (Tabla 2). En cuanto a la percepción de problemas en el trabajo o actividades cotidianas a causa de su salud física, se observó que un $65 \%$ manifestó haber hecho menos de lo que deseaba hacer (Tabla 3).

Sobre las percepciones durante las últimas cuatro semanas, a la pregunta "¿hasta qué punto su salud física o los problemas emocionales han dificultado sus actividades sociales habituales con la familia, los amigos, los vecinos u otras personas?" el $38 \%(n=15)$ manifestó "nada" y, en segundo lugar, se ubicó la respuesta "regular", con $23 \%(n=3)$. En cuanto a la presencia de dolor durante las últimas semanas, las opciones de respuesta "sí", "un poco" y "mucho" obtuvieron la misma frecuencia, $25 \%(\mathrm{n}=10)$, seguidas de la opción "moderado" con 18\% $(\mathrm{n}=7)$. En relación con la pregunta "¿hasta qué punto el dolor le ha dificultado su trabajo habitual (incluido el trabajo fuera de casa y las tareas domésticas)?", el 33\% $(n=13)$ seleccionó la opción "nada", seguido de la opción "bastante" con el 28\% (n=11). Por último, el $43 \%$ $(\mathrm{n}=17)$ de los participantes manifestó que "nunca" su salud física o problemas emocionales han dificultado sus actividades sociales (Tabla 4). En cuanto a los ítems relacionados con expectativas sobre su salud física, a la afirmación "mi salud es excelente" el 40\% seleccionó la respuesta "bastante falsa" (Tabla 5).

Las subescalas de función física, rol físico, dolor corporal, salud general y vitalidad presentaron valores promedio inferiores a 50. Por su parte, las subescalas como funcionamiento social, rol emocional y salud mental presentaron valores que superaron este valor: 67.81, 66.66 y 61.87, respectivamente (Tabla 6). En relación con los promedios por severidad de la enfermedad, se observaron algunos valores muy por debajo de 50 (promedio establecido para SF-36), como la vitalidad, con 39.24, y la función física, con 38.43, en los pacientes con EPOC severa o muy severa (Tabla 7).

\section{Discusión}

Este estudio evaluó la calidad de vida en adultos mayores con diagnóstico de EPOC, utilizando el SF-36 v2 en español para Colombia. Se encontró un claro impacto en la calidad de vida relacionada con la salud, especialmente en el componente físico. Esto mismo fue reportado en un estudio que mostró que pacientes con mayor severidad de la enfermedad presentaron, en este componente, valores alrededor de 37.4, aplicando el SF-36 (24). Este valor es similar al del presente estudio: 39.24. De igual forma, los puntajes de funcionamiento físico, vitalidad y salud general se asociaron significativamente con la alteración de la función respiratoria evaluada por el porcentaje de la $\mathrm{FEV}_{1}$, en donde los coeficientes de correlación fueron $0.44,0.25$ y 0.33 , respectivamente $(\mathrm{p}<0.01)(24)$.

Con respecto a las características sociodemográficas de los pacientes evaluados en el presente trabajo, los hallazgos son coherentes con lo reportados en otros estudios. En ellos, se ha definido la EPOC como una 
Tabla 1. Características sociodemográficas y clínicas de los participantes

\begin{tabular}{|c|c|c|}
\hline Características sociodemográficas & $\mathrm{n}$ & Porcentaje (\%) \\
\hline \multicolumn{3}{|l|}{ Sexo } \\
\hline Hombre & 28 & 70 \\
\hline Mujer & 12 & 30 \\
\hline \multicolumn{3}{|l|}{ Lugar de residencia } \\
\hline Bucaramanga & 33 & 82.5 \\
\hline Área metropolitana & 3 & 7.5 \\
\hline Otros departamento & 4 & 10 \\
\hline \multicolumn{3}{|l|}{ Escolaridad } \\
\hline Educación básica & 31 & 77.5 \\
\hline Técnica & 5 & 12.5 \\
\hline Profesional & 4 & 10 \\
\hline \multicolumn{3}{|l|}{ Características clínicas } \\
\hline \multicolumn{3}{|l|}{ Factores de riesgo para EPOC } \\
\hline Humo de biomasa & 8 & 20 \\
\hline Tabaquismo & 32 & 80 \\
\hline \multicolumn{3}{|l|}{ Severidad } \\
\hline Moderado (FEV1 50 - 80) & 20 & 50 \\
\hline Severo (FEV1 30 - 50) & 15 & 37.5 \\
\hline Muy severo (FEV1 < 30) & 5 & 12.5 \\
\hline \multicolumn{3}{|l|}{ Fuerza de músculos respiratorios } \\
\hline PImax $>80$ & 18 & 45 \\
\hline $\operatorname{PImax}<80$ & 22 & 55 \\
\hline PEmax $>80$ & 31 & 77.5 \\
\hline PEmax $<80$ & 9 & 22.5 \\
\hline
\end{tabular}

*EPOC: enfermedad pulmonar obstructiva crónica, $\mathrm{FEV}_{1}$ : volumen espiratorio forzado, PImax: presión inspiratoria máxima, PEmax: presión espiratoria máxima.

Fuente: elaboración propia de los autores 
Tabla 2. Respuesta a las preguntas relacionadas con cosas que podría hacer en un día normal, con tres opciones de respuesta

\begin{tabular}{|c|c|c|c|}
\hline Ítem & $\begin{array}{c}\text { Sí, me limita mu- } \\
\text { cho } \\
\%(n)\end{array}$ & $\begin{array}{l}\text { Sí, me limita un } \\
\text { poco } \\
\%(n)\end{array}$ & $\begin{array}{l}\text { No, no me limita } \\
\text { nada } \\
\%(n)\end{array}$ \\
\hline \multicolumn{4}{|l|}{ Su salud actual... } \\
\hline $\begin{array}{l}\text { ¿Le limita para hacer esfuerzos intensos, tales como } \\
\text { correr, levantar objetos pesados, o participar en } \\
\text { deportes agotadores? }\end{array}$ & $70(28)$ & $25(10)$ & $5(2)$ \\
\hline $\begin{array}{l}\text { ¿Le limita para hacer esfuerzos moderados, como } \\
\text { mover una mesa, pasar la aspiradora, jugar a los } \\
\text { bolos o caminar más de una hora? }\end{array}$ & $50(20)$ & $38(15)$ & $12(2)$ \\
\hline ¿Le limita para coger o llevar la bolsa de la compra? & $20(8)$ & $38(15)$ & $42(17)$ \\
\hline ¿Le limita para subir varios pisos por la escalera? & $73(29)$ & $20(8)$ & $7(3)$ \\
\hline ¿Le limita para subir un solo piso por la escalera? & $23(9)$ & $45(18)$ & $32(13)$ \\
\hline ¿Le limita para agacharse 0 arrodillarse? & $30(12)$ & $35(14)$ & $35(14)$ \\
\hline ¿Le limita para caminar un kilómetro o más & $42(17)$ & $35(14)$ & $23(9)$ \\
\hline $\begin{array}{l}\text { ¿Le limita para caminar varias manzanas (varios } \\
\text { centenares de metros)? }\end{array}$ & $40(16)$ & $35(14)$ & $25(10)$ \\
\hline $\begin{array}{l}\text { ¿Le limita para caminar una sola manzana (unos } \\
100 \text { metros)? }\end{array}$ & $25(10)$ & $25(10)$ & $50(20)$ \\
\hline ¿Le limita para bañarse o vestirse por sí mismo? & $7(3)$ & $30(12)$ & $63(25)$ \\
\hline
\end{tabular}

Fuente: elaboración propia de los autores

enfermedad que afecta a la población adulta mayor, con predominancia en el sexo masculino y residente en áreas urbanas (25).

En Colombia, se han aplicado varios de los instrumentos disponibles para evaluar la calidad de vida relacionada con la salud en pacientes con diferentes diagnósticos. Sin embargo, en razón de sus propiedades psicométricas, el SF-36 es uno de los instrumentos más ampliamente evaluados y utilizados para la medición de la calidad de vida en pacientes con enfermedades crónicas alrededor del mundo (26). Otros instrumentos han sido considerados para la evaluación de calidad de vida en pacientes con EPOC, pero el SF-36 representa el punto de referencia en cuanto a validez. Por lo tanto, su uso es recomendado para estos propósitos (27).
Para el caso particular de la EPOC en Colombia, la evaluación de la calidad de vida a través del SF-36 v2 solo está descrita en los trabajos realizados por Vinnacia (28). Ellos evaluaron 60 personas atendidas de manera ambulatoria con este diagnóstico en la ciudad de Medellín y encontraron que las subescalas físicas fueron las más afectadas, especialmente el rol físico (28). Estos resultados son similares a los encontrados en el presente estudio, donde se presentaron valores promedio inferiores a la media esperada para la población general. Es importante agregar que el deterioro de estas subescalas es más evidente en pacientes hospitalizados, debido al grado del compromiso de la función pulmonar por descompensación de su enfermedad. Así lo describen los hallazgos reportados en un estudio posterior del 
Tabla 3. Respuesta a las preguntas relacionadas con problemas en el trabajo o actividades cotidianas durante las 4 últimas semanas.

\begin{tabular}{lcc}
\hline Ítem & Sí & NO \\
$\%(\mathbf{n})$ & $\%(n)$ \\
\hline Problemas en el trabajo o actividades cotidianas, durante las 4 últimas semanas... & & \\
\hline $\begin{array}{l}\text { ¿Tuvo que reducir el tiempo dedicado al trabajo o a sus actividades cotidianas, a causa de su } \\
\text { salud física? }\end{array}$ & $53(21)$ & $47(19)$ \\
\hline $\begin{array}{l}\text { ¿Hizo menos de los que hubiera querido hacer, a causa de su salud física? } \\
\text { ¿Tuvo que dejar de hacer algunas tareas en su trabajo o sus actividades cotidianas (por ejem- } \\
\text { plo, le costó más de lo normal), a causa de su salud física? }\end{array}$ & $65(26)$ & $35(14)$ \\
\hline $\begin{array}{l}\text { ¿Tuvo que reducir el tiempo dedicado al trabajo o a sus actividades cotidianas, a causa de algún } \\
\text { problema emocional (como estar triste, deprimido o nervioso)? }\end{array}$ & $60(24)$ & $45(18)$ \\
\hline $\begin{array}{l}\text { ¿Tuvo que reducir el tiempo dedicado al trabajo o a sus actividades cotidianas, a causa de algún } \\
\text { problema emocional (como estar triste, deprimido o nervioso)? }\end{array}$ & $38(15)$ & $62(25)$ \\
\hline $\begin{array}{l}\text { ¿Hizo menos de lo que hubiera querido hacer, a causa de algún problema emocional (como } \\
\text { estar triste, deprimido o nervioso)? }\end{array}$ & $35(14)$ & $65(26)$ \\
\hline $\begin{array}{l}\text { ¿No hizo su trabajo o sus actividades cotidianas tan cuidadosamente como de costumbre, a } \\
\text { causa de algún problema emocional (como estar triste, deprimido o nervioso)? }\end{array}$ & $33(13)$ & $67(27)$ \\
\hline
\end{tabular}

Fuente: elaboración propia de los autores

Tabla 4. Respuesta a las preguntas relacionadas con las emociones y estados de ánimo durante las últimas 4 semanas.

\begin{tabular}{lcccccc}
\hline Ítem & $\begin{array}{l}\text { Siempre } \\
\%(\mathrm{n})\end{array}$ & $\begin{array}{l}\text { Casi siem- } \\
\text { pre } \%(\mathrm{n})\end{array}$ & $\begin{array}{l}\text { Muchas } \\
\text { veces } \%(\mathrm{n})\end{array}$ & $\begin{array}{l}\text { Algunas } \\
\text { veces } \%(\mathrm{n})\end{array}$ & $\begin{array}{l}\text { Solo al- } \\
\text { guna vez } \\
\text { (n) }\end{array}$ & $\begin{array}{l}\text { Nunca } \\
\%(\mathrm{n})\end{array}$ \\
\hline Durante las 4 últimas semanas... & & & & & & \\
\hline ¿Cuánto tiempo se sintió lleno de vida? & $8(3)$ & $13(5)$ & $18(7)$ & $35(14)$ & $13(5)$ & $15(6)$ \\
\hline ¿Cuánto tiempo estuvo nervioso? & $5(2)$ & $8(3)$ & $15(6)$ & $20(8)$ & $23(9)$ & $30(12)$ \\
\hline $\begin{array}{l}\text { ¿Cuánto tiempo se sintió tan bajo de moral } \\
\text { que nada podía animarle? }\end{array}$ & 0 & $3(1)$ & $15(6)$ & $35(14)$ & $13(5)$ & $35(14)$ \\
\hline ¿Cuánto tiempo se sintió calmado y tranquilo? & $13(5)$ & $28(11)$ & $15(6)$ & $35(14)$ & $5(2)$ & $5(2)$ \\
\hline ¿Cuánto tiempo tuvo mucha energía? & $15(6)$ & $18(7)$ & $3(1)$ & $35(14)$ & $20(8)$ & $10(4)$ \\
\hline ¿Cuánto tiempo se sintió desanimado y triste? & $8(3)$ & $5(2)$ & $10(4)$ & $45(18)$ & $13(5)$ & $20(8)$ \\
\hline ¿Cuánto tiempo se sintió agotado? & $10(4)$ & $20(8)$ & $23(9)$ & $25(10)$ & $8(3)$ & $15(6)$ \\
\hline ¿Cuánto tiempo se sintió feliz? & $15(6)$ & $18(7)$ & $15(6)$ & $35(14)$ & $10(4)$ & $8(3)$ \\
\hline ¿Cuánto tiempo se sintió cansado? & $8(3)$ & $28(11)$ & $30(12)$ & $20(8)$ & $5(2)$ & $10(4)$ \\
\hline
\end{tabular}

Fuente: elaboración propia de los autores 
Tabla 5. Respuesta a las preguntas relacionadas con la percepción de la propia salud.

\begin{tabular}{lccccc}
\hline Ítem & $\begin{array}{c}\text { Totalmente } \\
\text { cierto\% }(\mathbf{n})\end{array}$ & $\begin{array}{c}\text { Bastante } \\
\text { cierto\% }(\mathbf{n})\end{array}$ & $\begin{array}{c}\text { No lo sé } \\
\%(\mathbf{n})\end{array}$ & $\begin{array}{c}\text { Bastante } \\
\text { falso } \%(\mathbf{n})\end{array}$ & $\begin{array}{c}\text { Totalmente } \\
\text { falso\% }(\mathbf{n})\end{array}$ \\
\hline $\begin{array}{l}\text { Creo que me pongo enfermo más } \\
\text { fácilmente que otras personas }\end{array}$ & $8(3)$ & $18(7)$ & $23(9)$ & $33(13)$ & $20(8)$ \\
\hline Estoy tan sano como cualquiera & $8(3)$ & $18(7)$ & $20(8)$ & $35(14)$ & $20(8)$ \\
\hline Creo que mi salud va a empeorar & $5(2)$ & $10(4)$ & $30(12)$ & $38(15)$ & $18(7)$ \\
\hline Mi salud es excelente & $5(2)$ & $23(9)$ & $15(6)$ & $40(16)$ & $18(7)$ \\
\hline
\end{tabular}

Fuente: elaboración propia de los autores

Tabla 6. Promedio de las subescalas del SF-36 v2.

\begin{tabular}{lcc}
\hline Subescala & Media & DE \\
\hline Función física & 44 & 26.05 \\
\hline Rol físico & 44.38 & 44.03 \\
\hline Dolor corporal & 49.55 & 29.33 \\
\hline Salud general & 45.35 & 19.60 \\
\hline Vitalidad & 45.31 & 22.32 \\
\hline Funcionamiento social & 67.81 & 28.71 \\
\hline Rol emocional & 66.66 & 46.53 \\
\hline Salud mental & 61.87 & 20.99 \\
\hline
\end{tabular}

Fuente: elaboración propia de los autores

mismo autor, en el que se identificaron puntuaciones hasta de 8.1 para la dimensión de rol físico (29).

Con relación a la afectación de la subescala vitalidad, esta podría justificarse debido al grado de deterioro de la función pulmonar de estos pacientes, reflejado en un aumento de la fatiga durante la actividad física (30). Así lo describen en su más reciente estudio, en el cual los pacientes con EPOC alcanzaron un valor medio de 45.6 para la subescala descrita, muy por debajo del valor obtenido por el grupo de pacientes sanos, $76.25(\mathrm{p}=$ 0.004) (31). En este sentido, los pacientes del presente estudio mostraron valores similares en el componente físico, en especial en la subescala vitalidad, con un valor identificado de 45.31. Lo anterior es mediado por la similitud entre las características físicas y el estado de la enfermedad de los pacientes incluidos en ambos estudios. Asimismo, es importante destacar que los valores obtenidos en esta investigación son inferiores a los reportados en la población sana de Medellín, que presentó valores medios para todas las subescalas del SF-36 entre 75.8 y 79.5 (28).

Con respecto a las subescalas relacionadas con la salud mental general, los dos estudios realizados en Colombia muestran un deterioro marcado en pacientes hospitalizados. Por el contrario, en pacientes ambulatorios como los evaluados en nuestro estudio, 
Tabla 7. Evaluación de las subescalas por grupo de severidad de la enfermedad pulmonar obstructiva crónica.

\begin{tabular}{lcccc}
\hline Clasificación EPOC & Moderado & \multicolumn{3}{c}{$\begin{array}{c}\text { Severo / Muy se- } \\
\text { vero }\end{array}$} \\
\hline Subescalas & Media & DE & Media & DE \\
\hline Función física & 48.75 & 26.79 & 39.24 & 25.03 \\
\hline Rol físico & 40.00 & 43.97 & 48.75 & 44.77 \\
\hline Dolor corporal & 44.85 & 29.36 & 54.25 & 29.26 \\
\hline Salud general & 47.45 & 20.04 & 43.25 & 19.43 \\
\hline Vitalidad & 52.18 & 20.60 & 38.43 & 22.33 \\
\hline Funcionamiento social & 72.5 & 27.68 & 63.12 & 29.65 \\
\hline Rol emocional & 80 & 41.03 & 53.33 & 48.84 \\
\hline Salud mental & 69.5 & 19.45 & 54.25 & 20.08 \\
\hline
\end{tabular}

*EPOC: enfermedad pulmonar obstructiva crónica.

Fuente: elaboración propia de los autores

se encontraron valores cercanos a 50 y 79.5. Llama la atención, en este estudio, que una de las dimensiones con mayor puntaje fue la dimensión "funcionamiento social y rol emocional", con medias de 67.81 y 66.66 respectivamente. Estos hallazgos podrían justificarse por las características de la población aquí evaluada: los pacientes acudían de forma voluntaria a un programa de rehabilitación pulmonar y contaban con el apoyo económico y familiar para poder realizarlo. En este sentido, ha sido descrito el impacto que sobre la calidad de vida tienen los programas de rehabilitación. Fischer y colegas, en su estudio en pacientes con EPOC, encontraron una relación positiva entre asistir a este tipo de programas y la calidad de vida percibida, lo cual se evidencia en un cambio en la percepción de su enfermedad $(r=0.41$, $\mathrm{p}=0.002$ ) (32). De la misma manera, se ha descrito la relación que existe entre calidad de vida y apoyo social, dos factores que contribuyen a la adopción de conductas de autocuidado y control de los síntomas y progresión de la enfermedad (33-35).

Finalmente, es importante señalar, como limitación del presente trabajo, la falta de valores de referencia para la población colombiana, lo cual disminuye la posibilidad de interpretar de modo más contextualizado de los hallazgos de este estudio. A pesar de contar con el software, los valores utilizados por el mismo son calculados según promedios para población estadounidense. Se hace necesario, además, realizar otros estudios futuros, con una población mayor.

\section{Conclusiones}

Los pacientes con EPOC mostraron una disminución en la calidad de vida, frente a los valores de referencia para la población en general, en dos componentes: físico y mental. Se ratifica el impacto que tiene la enfermedad en todas las subescalas que componen este constructo, especialmente en el componente físico.

Con la dinámica del envejecimiento y el aumento de la expectativa de vida en países como Colombia, la gestión del cuidado en pro de la calidad de vida de los pacientes con enfermedades crónicas es un punto clave para la academia y el ámbito asistencial. Por esto, es necesario continuar con el desarrollo de investigaciones enfocadas en la evaluación de la calidad de vida relacionada con la salud, que identifiquen las dimensiones afectadas y orienten la planeación de intervenciones de enfermería. 
\begin{tabular}{l|l} 
Dedana Isabel Cáceres-Rivera, Enf., MSc., PhD. ,Zayne Milena Roa- \\
UNAB
\end{tabular} Robayo, FT., Esp., Mauricio Ariel Orozco-Levi, MD., Esp., PhD

\section{Consideraciones éticas}

Protección de personas y animales. Los autores declaran que en esta investigación no se han realizado experimentos en seres humanos ni en animales.

Confidencialidad de los datos. Los autores declaran que han seguido los protocolos de su centro de trabajo sobre la publicación de datos de pacientes.

Derecho a la privacidad y consentimiento informado. Los autores han obtenido el consentimiento de los pacientes referidos. Este documento obra en poder del autor de correspondencia.

\section{Conflicto de intereses}

Los autores declaran no tener ningún conflicto de interés.

\section{Financiación}

Proyecto INNOTORIO 744-2013 por COLCIENCIAS. Ref. Contrato 6566569337862013

\section{Referencias}

1. Pauwels RA, Buist AS, Calverley PM, Jenkins CR, Hurd SS. Global strategy for the diagnosis, management, and prevention of chronic obstructive pulmonary disease. NHLBI/WHO Global Initiative for Chronic Obstructive Lung Disease (GOLD) Workshop summary. Am J Respir Crit Care Med. 2001;163(5):1256-1276. doi:10.1164/ ajrccm.163.5.2101039

2. López MV, Montes de Oca M, Halbert RJ, Muiño A, Perez R, Tálamo C, et al. Sex-related differences in COPD in five Latin American cities: the PLATINO study. Eur Respir J. 2010;36(5):103441. doi:10.1183/09031936.00165409

3. Caballero A, Torres C, Jaramillo C, Bolivar F, Sanabria F, Osorio P, et al. Prevalence of COPD in Five Colombian Cities Situated at Low, Medium, and High Altitude (PREPOCOL Study). Chest. 2008;133(2):343-9. doi:10.1378/chest.07-1361

4. Srivastava K, Thakur D, Sharma S, Punekar YS. Systematic review of humanistic and economic burden of symptomatic chronic obstructive pulmonary disease. Pharmacoeconomics. 2015;33(5):467-88. doi:10.1007/s40273-015-
0252-4

5. Global Iniciative for Chronic Obstructive Lung Disease. Global Strategy for the Diagnosis, Managment and Prevention of COPD [Internet]. 2015 [citado 20 de julio de 2016]. Recuperado a partir de: http://www.goldcopd.org/uploads/ users/files/GOLD_Report_2015.pdf.

6. Rabe KF, Hurd S, Anzueto A, Barnes PJ, Buist SA, Calverley $\mathrm{P}$, et al. Global strategy for the diagnosis, management, and prevention of chronic obstructive pulmonary disease. GOLD Executive Summary. Am J Respir Crit Care Med. 2007;176(6): 532-55. doi:10.1164/rccm.200703$456 \mathrm{SO}$

7. Ries AL, Bauldoff GS, Carlin BW, Casaburi R, Emery CF, Mahler DA, et al. Pulmonary rehabilitation: joint ACCP/AACVPR evidencebased clinical practice guidelines. Chest. 2007;131(5 Suppl):4S-42S. doi:10.1378/ chest.06-2418

8. Nici L, Donner C, Wouters E, Zuwallack R, Ambrosino N, Bourbeau J, et al. American Thoracic Society/European Respiratory Society statement on pulmonary rehabilitation. Am J Respir Crit Care Med. 2006;173(12):1390-413. doi.10.1164/rccm.200508-1211ST

9. Díez J, Izquierdo JL, Rodríguez JM, Ramos P, Bellón JM, Molina J. Calidad de vida en la enfermedad pulmonar obstructiva crónica. Influencia del nivel de asistencia de los pacientes. Archivos de Bronconeumología. 2004;40(10):4317. doi.10.1016/S0300-2896(04)75567-2

10. Ramírez R. Calidad de vida y enfermedad pulmonar obstructiva crónica. Revista Ciencia de la Salud. 20017;5(1):90-100.

11. Van Manen JG, Bindels P, Dekker F, IJzermans C, Van der Zee J, Schadé E. Risk of depression in patients with chronic obstructive pulmonary disease and its determinants. Thorax. 2002;57(5):412-6. doi.10.1136/thorax.57.5.412

12. Whoqol Group. The World Health Organization Quality of life assessment (WHOQOL): Position paper from the World Health Organization. Soc Sci Med. 1995;41(10):1403-9. doi.10.1016/02779536(95)00112-K

13. Consiglio E, Belloso WH. Nuevos indicadores clínicos la calidad de vida relacionada con la 
salud. MEDICINA [Internet]. 2003 [citado 15 de febrero de 2013];63:172-8. Recuperado a partir de: http://www.scielo.org.ar/scielo.php?script=sci_ arttext\&pid=S0025-76802003000200014.

14. Taylor RM, Gibson F, Franck LS. A concept analysis of health-related quality of life in young people with chronic illness. J clin Nurs. 2008; 17(14):1823-33. doi.10.1111/j.13652702.2008.02379.x

15. Seidl M, Zannon CM. Quality of life and health: conceptual and methodological issues. Cad Saude Publica. 2004;20(2):580-8. doi.10.1590/S0102311X2004000200027

16. Urzúa AM, Caqueo A. Calidad de vida: Una revisión teórica del concepto. Ter Psicol. 2012;30(1):61-71. doi.org/10.4067/S0718 48082012000100006

17. Schwartzmann L. Calidad de vida relacionada con la salud: aspectos conceptuales. Cienc. enferm. 2003;9(2):9-21. doi.org/10.4067/S071795532003000200002

18. Vinaccia S, Orozco L.Aspectos psicosociales asociados con la calidad de vida de personas con enfermedades crónicas. Diversitas [Internet]. 2005 [citado 1 de noviembre de 2012];1(2):125-37. Recuperado a partir de: http://www.scielo.org.co/scielo.php?pid=S179499982005000200002\&script=sci_abstract\&tlng=es

19. Roales JG. Psicología de la salud: Aproximación histórica, conceptual y aplicaciones. Madrid, España: Ediciones Pirámide; 2004

20. Ware JE, Gandek B. Overview of the SF-36 Health Surveyand the International Quality of Life Assessment (IQOLA) Project. Journal of Clinical Epidemiology. 1998;51(11):903-12. doi. org/10.1016/S0895-4356(98)00081-X

21. Vilagut G, Ferrer M, Rajmil L, Rebollo P, Permanyer G, Quintana JM, et al. El cuestionario de salud SF-36 español: una década de experiencia y nuevos desarrollos. Gaceta sanitaria. 2005;19(2):135-50.

22. Maruish M. User's Manual for the SF-36v2 Health Survey. Tercera Edición. Lincold, EEUU: QualityMetric Incorporated; 2001.

23. Vilaguta G, Valderasa JM, Ferrer M, Garin O, López E, Alonsoab J. Interpretación de los cuestionarios de salud SF-36 y SF-12 en España: componentes físico y mental. Med Clin.
2008;130(19):726-35. doi.org/10.1157/13121076

24. Alonso J, Prieto L, Ferrer M, Vilagut G, Broquetas JM, Roca J, et al. Testing the Measurement Properties of the Spanish Version of the SF-36 Health Survey Among Male Patients with Chronic Obstructive Pulmonary Disease. J Clin Epidemiol. 1998;51(11):1087-94. doi. org/10.1016/S0895-4356(98)00100-0

25. Ramírez R. Calidad de vida relacionada con la salud como medida de resultados en salud: revisión sistemática de la literatura. Revista Colombiana de Cardiología. 2007;14(4):207-22.

26. Gutteling JJ, De Man RA, Busschbach JJ, Darlington AS. Overview of research on healthrelated quality of life in patients with chronic liver disease. Neth J Med [Internet]. 2007 [citado 1 de noviembre de 2012];65(7):227-34. Recuperado a partir de: http://www.njmonline.nl/getpdf. php?id $=547$

27. Paddison JS, Cafarella P, Frith P. Use of an Australian Quality of Life Tool in Patients with COPD. COPD. 2012;9(6):589-95. D. doi.10.310 9/15412555.2012.706666

28. Vinaccia S, Quiceno JM, Zapata C, Obesso S, Quintero DC. Calidad de vida relacionada con la salud y emociones negativas en pacientes con diagnóstico de enfermedad pulmonar obstructiva crónica (EPOC). Psicología desde el Caribe. 2006;18:89-108.

29. Vinaccia S, Quiceno JM. Calidad de Vida Relacionada con la Salud yFactores Psicológicos: Un Estudio desde la Enfermedad Pulmonar Obstructiva Crónica - EPOC. Ter Psicol. 2011;29(1):65-75. doi. org/10.4067/S0718-48082011000100007

30. Breslin, E. Percepción de la fatiga y la calidad de vida en pacientes con EPOC. Chest. 1998;114:958-64.

31. Antoniu SA, Petrescu E, Stanescu R, Anisie $\mathrm{E}$, Boiculese L. Impact of fatigue in patients with chronic obstructive pulmonary disease: results from an exploratory study. Ther Adv Respir Dis. 2016;10(1):26-33. doi. org/10.1177/1753465815617707

32. Fischer M, Scharloo M, Abbink J, Van 't HA, Van Ranst D, Rudolphus A, et al. The dynamics of illness perceptions: testing 
assumptions of Leventhal's common-sense model in a pulmonary rehabilitation setting. $\mathrm{Br}$ J Health Psychol. 2012;15(Pt4): 887-903. doi. org/10.1348/135910710X492693

33. Taylor S. Psicología de la Salud. México, D.F, México: McGraw-Hill; 2007.

34. Beneit PJ, Latorre JM. Representación mental de la enfermedad. Latorre. Psicología de la salud. 1994: 215-224.

35. Marks D, Murray M, Evans B, Willig C, Woodall C, Sykes CM. Psicología de la salud: Teoría, investigación y práctica. México D. F: Manual Moderno; 2008. 